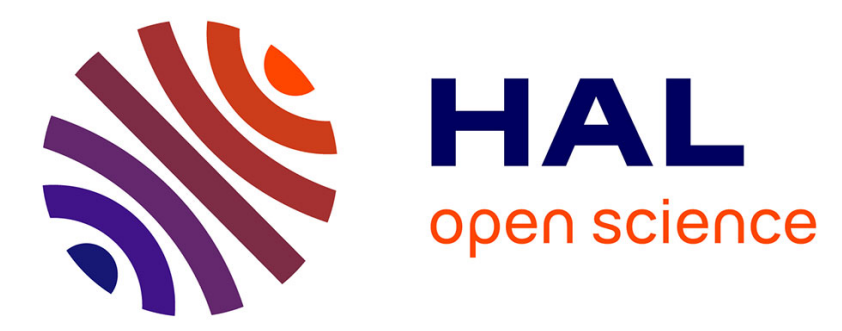

\title{
Valeur et travail chez Destutt de Tracy
}

Jean Magnan de Bornier

\section{To cite this version:}

Jean Magnan de Bornier. Valeur et travail chez Destutt de Tracy. 2008. halshs-00278528

\section{HAL Id: halshs-00278528 \\ https://shs.hal.science/halshs-00278528}

Preprint submitted on 13 May 2008

HAL is a multi-disciplinary open access archive for the deposit and dissemination of scientific research documents, whether they are published or not. The documents may come from teaching and research institutions in France or abroad, or from public or private research centers.
L'archive ouverte pluridisciplinaire HAL, est destinée au dépôt et à la diffusion de documents scientifiques de niveau recherche, publiés ou non, émanant des établissements d'enseignement et de recherche français ou étrangers, des laboratoires publics ou privés. 


\section{GREQAM}

Groupement de Recherche en Economie Quantitative d'Aix-Marseille - UMR-CNRS 6579

Ecole des Hautes Etudes en Sciences Sociales Universités d'Aix-Marseille II et III

\title{
Valeur et travail chez Destutt de Tracy
}

\author{
Jean Magnan de Bornier
}

Mars 2008

Document de Travail $n^{\circ}$ 2008-16 


\title{
Valeur et travail chez Destutt de Tracy
}

\author{
Jean MAGNAN de BORNIER \\ Université Paul Cézanne à Aix-Marseille et GREQAM \\ email: jean.magnanb@univ-cezanne.fr
}

15-19 Allée Claude Forbin, 13627 Aix-en-Provence Cedex 1 FRANCE

\section{9 mars 2008}

\begin{abstract}
Résumé
On examine la théorie de la valeur de Destutt de Tracy et ses rapports avec le travail. Cet auteur propose une théorie originale comportant deux valeurs, l'une naturelle et l'autre conventionnelle. Seule la première est reliée au travail, qui est conçu comme toute activité productive et la seule origine de toute production. La théorie des deux valeurs amène finalement à des contradictions insurmontables, dues probablement à la tentative de déduire l'économie politique de la philosophie des idéologistes.
\end{abstract}

\section{Summary}

Destutt de Tracy's value theory is examined here in its relations to labor. This theory is original in that it has two concepts of value, one being naural, the other, conventional. Only the first of these is linked to labor, deemed to be any productive activity and the sole origin of any production. This two-value theory leads to insuperable contradictions, probably to be explained by the author's aim to deduce political economy from the idéologistes' philosophy.

\section{Table des matières}

1 Introduction 2

$2 \quad$ Un diagnostic largement partagé depuis Ricardo 3

3 Le sens et la place du travail chez Destutt 5

4 Les deux valeurs 8

$5 \quad$ Valeur, travail, quel concept mesure l'autre? $\quad 9$

6 Conclusion 11

Mots-clés

Destutt de Tracy, Idéologie, valeur, travail 


\section{Introduction}

\section{Introduction}

Destutt est un personnage à facettes multiples. Ancien colonel de cavalerie, député de la noblesse aux États généraux de 1789, il vote l'abolition des privilèges lors de la nuit du 4 août, puis est maréchal de camp de La Fayette quand la Constituante est dissolue en 1792. En 1793 et 94 il passe onze mois en prison (c'est la période de la mort de Condorcet), où il reprend la lecture les philosophes, dont particulièrement Locke et Condillac. Avec Cabanis il développe dès 1792 une activité scientifique; avec ce même Cabanis il fondera en 1795 la Société des Idéologues, dont l'objet est l'idéologie ou «science des idées», proche des idées de Condorcet et qui comptera Jean-Baptiste Say parmi ses membres et soutiens ${ }^{1}$. Sénateur et académicien ${ }^{2}$, il n'en a pas moins connu dès la Restauration une perte d'influence, auprès du pouvoir, des idées qu'il défendait, trop proches des Lumières et de la Révolution; c'est la philosophie de Victor Cousin, non plus celle des idéologues, qui représente alors la «pensée officielle».

Pour Destutt de Tracy, le propos sur la société intervient après un long cheminement, et comme déduction de vérités premières (ce que Cabanis nommait plus volontiers des «faits généraux»), de causes au-delà desquelles il serait impossible de remonter. Ainsi son ouvrage d'économie n'est que le quatrième et dernier tome - d'ailleurs incomplet - des Éléments d'Idéologie; les trois premiers sont L'idéologie proprement dite (1801), La grammaire (1803) puis La logique (1805); le quatrième tome s'appelait Traité de la volonté lors de sa première publication (1815), avant d'être renommé en 1823 Traité d'économie politique (on le désignera par Traité ci-après), ce qui correspond beaucoup mieux à son contenu (Thomas Jefferson avait supervisé la traduction américaine du Traité de la volonté, qui est apparue en 1818 sous le titre Treatise on Political Economy). L'écriture du Traité a été précédée d'une œuvre indépendante, mais contenant de nombreuses pages consacrées à l'économie, dont certaines seront d'ailleurs reprises sans modification dans le Traité, le Commentaire sur l'esprit des lois de Montesquieu (1819). Ces deux textes constituent les principales sources permettant d'apprécier la pensée économique de Destutt.

1 Stendhal a été un lecteur d'un enthousiasme absolu de Destutt, comme en témoignent de nombreuses lettres adressées à sa sœur Pauline; par exemple, «Je ne puis laisser un vide sans te parler de l'Idéologie, qui, t'apprenant à ne point faire de vœux contradictoires, te met sur la route du bonheur. Lis et relis ce livre sublime» Stendhal (1962) (p.247)

2 Il a été membre de l'Institut puis élu à l'Académie française en 1808. 


\section{Un diagnostic largement partagé depuis Ricardo}

Dans les Éléments d'Idéologie Destutt part de ce qu'il considère comme les données les plus basiques et les plus certaines: les sensations, toucher, vue, etc. Quelles connaissances, quelles certitudes peut-on en tirer? C'est ce qu'il cherche à connaître; les principales chaînes de déduction lui permettront de penser que l'être sentant existe (le je), puis en allant plus loin, qu'autrui existe aussi. La voie est ouverte - après d'autres détours - pour étudier la société, puisque l'être et sa multiplicité sont alors des faits démontrés.

Nous nous concentrerons ici sur les relations entre valeur et travail chez cet auteur trop peu connu, mais il importe de préciser que de nombreux autres domaines de sa pensée méritent lecture et analyse; sa théorie de la distribution, de la condition des pauvres, de la population en sont des aspects notables mais qu'on ne touchera pas.

\section{Un diagnostic largement partagé depuis Ricardo}

S'il y a assez peu de commentateurs de Destutt, presque tous ont semblé proposer, à des degrés divers, l'idée qu'il avait une théorie de la valeur qui le plaçait plutôt du côté de Ricardo que de celui de Say, dont il était pourtant proche. Ce diagnostic s'établit, comme c'est souvent le cas pour des auteurs peu lus, par imitation autant que par un examen où l'on procéderait tabula rasa. Diagnostic assez peu crédible au fond, et dont on montrera les limites. Tout commence avec Ricardo.

Dans la troisième édition de ses Principes (Ricardo (1951)), il rajoute au chapitre XX, où il discute de valeur et de richesses (Value and Riches, Their Distinctive Properties), un argument contre Say, sous forme d'une citation du Traité de Destutt:

A franc is not a measure of value for any thing, but for a quantity of the same metal of which francs are made, unless francs, and the thing to be measured, can be referred to some other measure which is common to both. This, I think, they can be, for they are both the result of labour; and, therefore, labour is a common measure, by which their real as well as their relative value may be estimated. This also, I am happy to say, appears to be M. Destutt de Tracy's opinion. He says, "as it is certain that our physical and moral faculties are alone our original riches, the employment of those faculties, labour of some kind, is our only original treasure, and that it is always from this employment, that all those things are created which we call riches, those which are the most necessary, as well as those which are the most purely agreeable. It is certain too, that all those things only represent the labour which has created them, and if they have a value, or even two distinct values, 


\section{Un diagnostic largement partagé depuis Ricardo}

they can only derive them from that of the labour from which they emanate." (pp. $384-85)^{3}$

La position de Ricardo est cependant ambiguë: il semble utiliser Destutt comme argument contre Say en ce qui concerne la notion de mesure ${ }^{4}$, plus qu'affirmer que Destutt soutiendrait une théorie de la valeur proche de la sienne propre. Il écrit d'ailleurs en note, pour accompagner cette citation:

In this work M. de Tracy has given a useful and an able treatise on the general principles of Political Economy, and I am sorry to be obliged to add, that he supports, by his authority, the definitions which M. Say has given of the words "value," "riches," and "utility." (ibid.)

Cependant Ricardo a sans doute marqué les esprits. Les quelques spécialistes se sont rangés, d'une manière plus ou moins ouverte, à l'idée que sa théorie de la valeur peut être rangée dans la catégorie «valeur-travail».

À l'aube du vingtième siècle, Allix (1912) pouvait constater (p. 493):

Malgré sa fidélité à suivre, en général, J.-B. Say, Destutt se sépare de lui sur la question de la valeur. Il adopte la théorie, déjà exposée par Smith et qui rendra plus tard célèbre le nom de Ricardo, que toute valeur vient du travail.

Plus récemment, dans son étude par ailleurs remarquable sur Destutt, Kennedy (1978) ne dévie pas de l'opinion établie:

Tracy's labor theory might have led him to advocate a radical redistribution of wealth, and this would have placed him in the ranks of distinguished nineteenth-century socialists who sought to alleviate industrial misery. (p. 198)

De même Klein (1985) affirmait récemment:

Tracy's notion of necessary value, based on the costs of production, does not find a significant role in his dynamic conception of economics. [.. . ] Lacking the marginalist insights that spring from catallactic dynamics, he does not make the proper integration of costs into an equilibrium theory of market price; yet, he clings to a cost notion of value (the "natural and necessary value") despite its deficiency. (p. 67)

On peut d'ailleurs trouver chez Destutt des passages qui suggèrent, encore plus fortement que celui cité par Ricardo, une théorie de la valeur-travail; dans le Commentaire sur L'esprit des lois Destutt semble très explicite:

Les philosophes, appelés exclusivement économistes, n'avaient donc pas assez observé la nature de l'homme et spécialement sa nature intellectuelle : ils n'avaient pas vu que dans nos facultés et dans l'emploi qu'en fait notre volonté, consistent tous

${ }^{3}$ Ricardo indique comme source la page 99 du Traité, alors que ce passage figure aux pages 36-37.

4 Schumpeter a bien perçu ce point, et note que Ricardo se contredit à cet égard Schumpeter (1954). 


\section{Le sens et la place du travail chez Destutt}

nos trésors ; et que cet emploi, le travail, est la seule richesse qui ait par elle-même une valeur primitive, naturelle et nécessaire, qu'elle communique à toutes les choses auxquelles elle est appliquée, et qui n'en sauraient avoir d'autre Destutt de Tracy (1819)

La lecture de ces quelques passages laisse imaginer, plus qu'elle ne démontre, une relation forte entre valeur et travail chez Destutt; mais faute d'une analyse poussée, cette relation n'est pas précisée; essayons-nous à cette tâche.

\section{Le sens et la place du travail chez Destutt}

Le travail chez Destutt se définit comme l'action de produire, et il est seul à avoir cette capacité. Cette double affirmation n'est pas banale et mérite en conséquence quelques développements.

La définition du travail chez Destutt est très générale et indépendante de toute contingence sociale; le travail, c'est purement et simplement l'emploi par chaque être de ses facultés: en effet, l'homme

ne peut (...) pourvoir [à des besoins sans cesse renaissants] que par l'emploi de ces

facultés, de ses moyens ; et si cet emploi, son travail, cessait pendant un certain

temps de faire face à ses besoins, son existence finirait. (Destutt de Tracy (1815),

p. 36)

Travailler, c'est employer ses facultés ou ses moyens en vue de pourvoir à ses besoins.

L'entrepreneur, qui s'emploie lui-même, est un travailleur au même titre que le salarié, même s'il mélange des capitaux à son travail. Cette définition évoque le travail tel que le voit Locke dans son Traité du gouvernement civil, (au chapitre V «De la propriété») un ouvrage que cependant Destutt ne cite nulle part ${ }^{5}$, quoiqu'il l'aie certainement lu. Le travail est une activité qui, même avant la mise en place des conventions, dans l'état de nature, existe déjà, et qui ne change pas de nature après leur établissement ${ }^{6}$.

Concernant la seconde affirmation et la place du travail et sa productivité, Destutt se range explicitement dans le camp de Smith et Say (selon lui), qu'il oppose aux physiocrates. C'est sur le thème de la distinction entre ce qui est productif ou improductif que se situe le point de départ de ce débat:

5 Il y a des références à l'Essai sur l'entendement humain dans les volumes i et iii des Éléménts d'Idéologie.

6 On notera que Marx, dans les propos acides qu'il consacre à Destutt dans Théories sur la plus-value, n'a pas perçu cette particularité de sa définition du travail; il interprète ses vues à la lumière (si on peut dire) que le travailleur est le prolétaire. 


\section{Le sens et la place du travail chez Destutt}

Le savant et judicieux écossais, Adam Smith, a bien vu que le travail est notre seul trésor, et que tout ce qui compose la masse des richesses d'un particulier ou d'une société, n'est autre chose que du travail accumulé, parce qu'il n'a pas été consommé aussitôt que produit. Il a reconnu que tout travail, qui ajoute à cette masse de richesses plus que n'en consomme celui qui l'exécute, doit être appelé productif, et qu'il n'est improductif que dans le cas contraire (Destutt de Tracy (1819), p.284)

Plus loin:

M. Say, ancien membre du tribunat français, qui est sans contredit l'auteur du meilleur livre d'économie politique qui ait encore été fait, et qui a écrit long-temps après Smith, voit bien comme lui que l'emploi de nos facultés est la source de toutes nos richesses, et que lui seul est la cause de la valeur nécessaire de tout ce qui en a une. (Destutt de Tracy (1819), p.285)

Mais ni Say ni Smith ne vont assez loin dans leur analyse de la place du travail, qui selon Destutt est le seul facteur de production. Dans le Commentaire, il règle rapidement - suivant en cela Smith - le compte des machines ou du capital, qui ne sont que du «travail accumulé», pour consacrer plus de place à la terre; il montre d'abord qu'il n'y a pas de différence, du point de vue de la logique économique, entre une terre et une machine. Ainsi, Smith

croit voir encore dans la rente de la terre autre chose que ce qu'il appelle les profits d'un capital[...] Il dit bien qu'une ferme améliorée peut être regardée sous le même point de vue, que ces machines utiles qui facilitent le travail ; mais il n'ose dire nettement, ce qui est pourtant vrai, qu'un champ est un outil comme un autre, et que son fermage est tout-à-fait la même chose, que le loyer d'une machine ou l'intérêt d'une somme prêtée. (Destutt de Tracy (1819), p.285-6)

Et Say est accusé de tomber dans la même erreur:

M. Say prononce sans hésiter, liv. I, chap. 5, qu'un fonds de terre n'est qu'une machine. Néanmoins, entraîné par l'autorité de ses prédécesseurs, qu'il a si souvent corrigés et surpassés, ou peut-être dominé seulement par l'empire de l'habitude et de je ne sais quel prestige, M Say lui-même revient ensuite à se laisser éblouir par l'illusion, qu'il a détruite le plus complétement possible. Il s'obstine à regarder un fonds de terre comme un bien d'une nature tout-à-fait particulière, son service productif comme autre chose que l'utilité d'un outil, et son fermage comme différent du loyer d'un capital prêté.(Destutt de Tracy (1819), p.287-8)

L'erreur de Say serait donc d'avoir proposé la théorie des trois facteurs avec leurs rémunérations différenciées, que Destutt énumère ainsi «1) des profits de travail ou salaires; 2) des profits de capitaux, qui semblent une chose différente des premiers; 3) des profits de fonds de terre, qui paraissent encore un élément d'un tout autre genre.»(ibid.) 


\section{Le sens et la place du travail chez Destutt}

Cette multiplicité des types de revenus ne peut qu'avoir des inconvénients, «[o]n ne sait comment déterminer le prix naturel et nécessaire de chaque chose. Il y en a toujours une portion dont on ne voit pas la cause ... Tout est embrouillé et sophistiqué dès le principe, et on ne peut plus se faire, sur tous ces objets, que des opinions arbitraires et incohérentes.» (pp. 289-90)

Destutt, on le voit dans ces phrases, veut ce qu'on pourrait appeler une théorie «unitaire» de la production: un seul facteur, un seul type de revenu, qu'on appelle «salaire», quoique Destutt considère que c'est à tort ${ }^{7}$

Dans le Traité, Destutt discutera de la séparation en classes, d'abord pour la nier au chapitre VIII «De la distribution de nos richesses entre les individus», puis au contraire l'acceptant totalement au chapitre X qui poursuit sur les inégalités et la justice:

Rappelons-nous que la société est partagée en deux grandes classes: celles des hommes qui, sans voir aucune avance, travaillent moyennant un salaire, et celle des hommes qui les emploient. (Destutt de Tracy (1815), p.200)

Mais cette reconnaissance ne signifie nullement que les entrepreneurs ne travaillent pas, qu'ils ne sont pas des agents «productifs». Le travail n'est pas le fait exclusif de la première classe, même si la seconde comprend, en plus des entrepreneurs, ceux «qui vivent de leurs revenus sans travail»(ibid.). La distinction des classes, qui mène à des développements dignes d'intérêt dans les chapitres VIII à X, ne joue aucun rôle dans la théorie de la valeur que Destutt présente dans l'introduction du Traité, puis dans ses chapitres II et III.

Finalement on voit que Destutt propose un traitement du travail et des facteurs de production qui, se réclamant de Smith et Say pour mieux réfuter les physiocrates, ne correspond cependant réellement à l'approche d'aucun des deux auteurs qu'il revendique.

De cette conception purement technique ou naturaliste du travail, on tire deux conséquences assez évidentes en matière de valeur:

- Une théorie de la valeur qui serait fondée sur le coût monétaire du travail (le salaire) ne peut pas sortir de cette conception, dans la mesure où le travail peut, selon le statut du travailleur, avoir un coût monétaire - si c'est un employé - ou n'en pas avoir.

- Toute valeur qu'on reconnaît aux biens tire son origine, a sa cause, dans le travail, et cela indépendamment de la mesure de cette valeur.

7 «On dit communément que les bénéfices de l'entrepreneur, mal à propos appelés salaires, puisque personne ne lui a rien promis ...»(Destutt de Tracy (1815), p.102) 


\section{Les deux valeurs}

\section{Les deux valeurs}

Dans le chapitre III du Traité, Destutt définit l'utilité et son rapport avec la valeur; son utilité est tout à fait subjective, elle est équivalente à «la vivacité de nos désirs»(Destutt de Tracy (1815), p. 90). C'est à travers les échanges que la valeur des biens utiles est déterminée:

Dans l'état de société, qui n'est qu'une suite continuelle d'échanges, c'est ainsi que se déterminent les valeurs de tous les produits de notre industrie. Cette fixation, sans doute, n'est pas toujours fondée sur de bien bonnes raisons ; nous sommes souvent de très mauvais appréciateurs du vrai mérite des choses mais enfin, sous le rapport de la richesse, elles n'en ont pas moins la valeur que leur assigne l'opinion générale. (ibid.)

Pourtant on n'en restera pas là, parce qu'en réalité tous les biens ont deux valeurs ${ }^{8}$ :

Maintenant, ces biens ont tous parmi nous une valeur déterminée et fixe jusqu'à un certain point; ils en ont même toujours deux; l'une est celle des sacrifices que nous coûte leur acquisition; l'autre, celle des avantages que nous procure leur possession. (Destutt de Tracy (1815), p. 35)

Ces deux valeurs sont qualifiées, pour la première, de naturelle et nécessaire et pour la seconde, de conventionnelle et éventuelle.

La valeur nécessaire, qui apparaît à première vue comme le coût de production, est définie plus loin comme «la somme des besoins indispensables dont la satisfaction est nécessaire à l'existence de celui qui exécute ce travail, pendant le temps qu'il l'exécute»(p.92), n'est donc pas une quantité de travail. C'est une valeur qui doit être présente pour que la production puisse avoir lieu, elle concerne les biens nécessaires à la survie du travailleur, et cela explique que cette valeur est «naturelle». Cette définition de la valeur nécessaire permet à Destutt de préciser la relation entre les deux valeurs: si la valeur conventionnelle est inférieure à la valeur naturelle, il faut que le travailleur «se livre à une autre industrie, ou il s'éteint»; si elle lui est juste égale, «il subsiste avec peine», et si elle lui est supérieure, «il s'enrichit».(ibid.)

La valeur conventionnelle, quant à elle, semble avoir un statut supérieur, un statut de vérité:

Dans tous les cas, cette valeur conventionnelle et vénale est la véritable sous le rapport de la richesse; elle est la vraie mesure de l'utilité de la production, puisqu'elle en fixe le prix. (Destutt de Tracy (1815), p. 92)

8 Dans le Commentaire, le raisonnement qu'on décrit ici est mené en utilisant le terme «prix» au lieu de «valeur». 
Valeur, travail, quel concept mesure l'autre?

En effet Destutt propose une théorie de la fixation des prix qui fait intervenir divers éléments, dont l'utilité occupe la première place. Mais «cette valeur de convention, ce prix vénal»a d'autres déterminants que «l'estime qu'on fait»; elle varie suivant les «besoins et les moyens» des différents agents: producteur et consommateur, acheteur et vendeur, et aussi suivant leur nombre. Ainsi, les ingrédients d'une théorie qu'on jugerait assez complète aujourd'hui des prix de marché sont présents: les préférences des agents (l'estime), les coûts (les besoins), les revenus (les moyens), la structure des marchés (le nombre des acheteurs et des vendeurs). S'il semble à Destutt impossible de développer une description complète de la fixation des prix, parce que ces divers éléments sont trop difficiles à analyser, il en a proposé une architecture générale.

De plus, la valeur conventionnelle a une mesure sans ambiguïté: c'est la monnaie, l'or et l'argent (Destutt de Tracy (1815), p. 140).

On est tenté, évidemment, de résumer la théorie des deux valeurs en disant que l'une est un prix et l'autre un coût; mais ce serait masquer l'originalité du concept de valeur nécessaire, ce serait masquer aussi certaines de ses particularités, liées à la question importante de la mesure, qu'on se propose maintenant d'exposer.

\section{Valeur, travail, quel concept mesure l'autre?}

Il semble paradoxal que la valeur nécessaire donne lieu à plus de développements dans l'introduction du Traité que dans le corps du développement, particulièrement au chapitre consacré à la valeur. En effet, dans cette introduction, la relation entre travail et valeur est précisée dans une direction qui ne pouvait pas plaire à Ricardo, avec l'affirmation suivante (cette phrase suit directement la citation de Ricardo):

Le travail lui-même a donc une valeur; il en a même deux différentes (...) oui, le travail a ces deux valeurs, l'une naturelle et nécessaire, l'autre plus ou moins conventionnelle et nécessaire (Destutt de Tracy (1815), p. 36)

La mesure naturelle du travail, c'est la «masse des besoins»; quoiqu'on ne sache pas comment la mesure de cette masse est effectuée, elle représente ce qui est nécessaire à la survie, celle de «ce que ce travail coûte inévitablement»(p. 37). La valeur conventionnelle du travail est «celle de ce qu'il produit», et la convention à laquelle on doit se référer est soit le contrat de travail, soit le profit réalisé; en effet, si le travailleur est un salarié, la convention a été «faite avant le travail exécuté», s'il s'agit d'un marchand ou fabricant, «elle ne s'opère qu'après le travail fait et parfait» (ibid). 


\section{Valeur, travail, quel concept mesure l'autre?}

L'idée de valeur du travail reflète donc une variété d'objets: pour la valeur conventionnelle, ce sera tout type de revenu, quelque soit son mode de détermination; et nulle part Destutt ne précise si la valeur naturelle est un coût monétaire: tentons de poursuivre son raisonnement.

En reprenant ce que Destutt dit du travail, ne pourrait-on affirmer que la «masse des besoins» que constitue la valeur nécessaire a sans doute une valeur, sinon elle ne pourrait transmettre une valeur, elle en a même deux, etc... Ce raisonnement permettrait de réduire in finum tous biens de toute sorte en quantités de travail; mais une comptabilité en travail serait sans doute embarrassante dans la représentation que Destutt se fait de la valeur; en effet «aucun être ne peut communiquer une propriété qu'il n'a pas»(p. 36); si on admettait que le travail se mesure ultimement en travail, il ne pourrait pas communiquer de la valeur, et la valeur serait un objet inexpliqué.

Telle est peut-être une raison pour laquelle la valeur nécessaire ne fera pas l'objet, dans le corps du Traité, de développements supplémentaires, alors que la valeur conventionnelle sera approfondie. Mais il y a sans doute une autre raison: l'introduction du Traité est une partie où les trois premiers volumes de l'Idéologie sont survolés, et où est fait le lien entre les propositions philosophiques qu'ils contiennent et le livre qui s'ouvre. Il est essentiel, dans la grande vision unitaire de Destutt, qui part de la sensation comme principe explicatif quasiment unique, que l'économie politique, la science sociale entière même, puisse être déduite des premiers principes - et la raison d'être de cette longue introduction est de faire ce lien, de montrer pourquoi l'économie dépend d'une bonne idéologie. Trois principes généraux sont ainsi désignés, dans l'introduction, comme des principes premiers auxquels l'analyse de la valeur devra se plier, trois principes qu'on nommera ${ }^{9}$ : un principe de mesure, un principe d'antériorité du naturel, un principe de communication.

\section{- Principe de mesure}

En se référant à ce principe, Destutt renvoie aux tomes 1 et 3 , en particulier: «mesurer une chose quelconque, c'est la comparer à une quantité déterminée de cette même chose que l'on prend pour terme de comparaison, pour unité.» (Destutt de Tracy (1801), p. 186). C'est bien cette idée de Destutt que Ricardo tentera d'exploiter.

\footnotetext{
9 Appellations de l'auteur du présent texte.
} 


\section{Conclusion}

\section{- Principe d'antériorité du naturel}

Rien ne peut exister dans le monde des conventions, de l'artifice, qui ne tirerait pas son existence de quelque chose de naturel; pour la valeur: «puisque nous avons l'idée de valeur, et puisqu'il existe parmi nous des valeurs artificielles et conventionnelles, il fallait qu'il y eût quelque part une valeur naturelle et nécessaire.» (Destutt de Tracy (1815), p. 39) ${ }^{10}$

\section{- Principe de communication}

Selon ce principe, «aucun être ne peut communiquer une propriété qu'il n'a pas» (p. 36). Si une valeur est communiquée par le travail, c'est que le travail a une valeur.

L'ensemble de ces principes mène, nous semble-t-il, à une impasse si on pousse l'analyse assez loin; elle amène à faire de la valeur naturelle un objet inexplicable, car on aura toujours besoin de remonter la chaîne de valeur; s'en tenir aux valeurs conventionnelles permet de sortir de l'impasse, mais coupe le lien avec les fondements philosophiques de l'économie politique.

\section{Conclusion}

À la question «Destutt propose-t-il une théorie de la valeur-travail?», on ne pourra répondre qu'avec précaution; s'il s'agit d'une valeur-travail du type de celle de Ricardo ou de Marx, la réponse sera négative. Mais on peut en fait définir plusieurs approches de la valeur-travail; selon Vaughn (1978) qui traite du cas de Locke, on peut donner trois sens différents à cette notion:

There are three possible meanings of a labor theory of value that are relevant to

Locke's writings: a labor theory of value may identify labor as the source of use-value or utility (...), it may attempt to explain the determination of relative prices (...), or it may claim that labor provides the only justifiable claim to receiving the exchange value of the goods it produces. (p. 311)

La théorie de Destutt semble appartenir au premier de ces types, à condition évidemment que le terme «travail» soit compris dans le même sens que notre auteur.

Le projet que mène Destutt de fonder les sciences sociales sur des fondements «idéologiques» - c'est-à-dire métaphysiques - le pousse à développer une

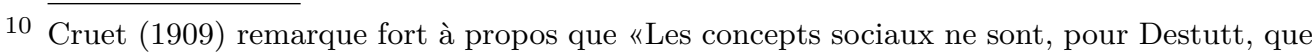
le prolongement dans la société des traits distinctifs de la nature humaine.» (p. 72) 


\section{Conclusion}

théorie de la valeur excessivement compliquée et finalement contradictoire. Il est intéressant de le comparer sur ce point à Say; ce dernier, on le sait, et malgré ses liens avec les idéologistes, n'entend faire entrer aucune philosophie dans son $\operatorname{propos}^{11}$, et ceci s'applique évidemment à sa théorie de la valeur, une valeur uniquement «conventionnelle» bien entendu. L'approche de Say, non encombrée de métaphysique, permet sans doute un certain progrès et une certaine reconnaissance. Elle lui permet d'avancer et dans une certaine mesure de renverser le lien de causalité entre la valeur des produits et celle des facteurs par rapport àl'analyse smithienne,ce que Destutt n'aurait pu développer, du seul fait de sa dualité de la valeur et des principes mentionnés ci-dessus.

D'une certaine manière, Destutt est un témoignage supplémentaire d'une des propriétés de la philosophie économique: si la construction de propos philosophiques sur un corpus constitué de théorie économique est une activité relativement courante (on ne veut pas dire facile), la fabrication réussie d'un tel corpus à partir d'une philosophie n'est-elle pas beaucoup plus rare et délicate, peut-être même impossible?

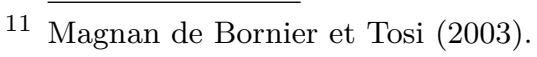




\section{Conclusion}

\section{Références}

Allix, E. (1912). Destutt de Tracy, économiste. Revue d'Économie Politique, pages $424-51$.

Cruet, J. (1909). La philosophie morale et sociale de Destutt de Tracy (1754-1836). Allard, Tours.

Destutt de Tracy, A. (1819). Commentaire sur "L'esprit des lois" de Montesquieu. Delaunay Mongié ainé, Paris.

Destutt de Tracy, A. (1803). Éléments d'idéologie: grammaire. Didot, Paris.

Destutt de Tracy, A. (1801). Éléments d'idéologie: idéologie proprement dite. Didot, Paris.

Destutt de Tracy, A. (1805). Éléments d'idéologie: logique. Didot, Paris.

Destutt de Tracy, A. (1815). Éléments d'idéologie: Traité de la volonté et de ses effets. Courcier.Quatrième partie des Éléments d'idéologie. Réédité sous le titre "Traité d'Économie Politique" en 1823.

Magnan de Bornier, J. et Tosi, G. (2003). Jean-Baptiste Say, Nouveaux regards sur son œuvre, Chapitre: La méthode de Jean-Baptiste Say. Economica. Ouvrage coordonné par Jean-Pierre Potier et André Tiran.

Kennedy, E. (1978). Destutt de Tracy and the Origins of "Ideology". The American Philosophical Society, Philadelphia.

Klein, D. (1985). Deductive Economic Methodology in the French Enlightenment: Condillac and Destutt de Tracy. History of Political Economy, pages $51-71$.

Picavet, F. (1891). Les Idéologues. Alcan, Paris.

Ricardo, D. (1951). On the Principles of Political Economy and Taxation. Cambridge University Press. Works and Correspondence of David Ricardo, volume 1, edited by Piero Sraffa with the collaboration of Maurice Dobb.

Schumpeter, J. A. (1954). History of Economic Analysis. Oxford University Press, New-York.

Stendhal (1962). Correspondance, volume 1. NRF La Pléiade, Paris.Édition établie par et annotée par Henri Martineau et V. del Litto.

Vaughn, K. (1978). John locke and the labor theory of value. Journal of Libertarian Studies, 2(4):311-326. 